\title{
Alleviating an Increasingly Burdened Healthcare System with Telemedicine: Anterior Segment
}

Karam A. Alawa $\cdot$ Christopher S. Sales

Received: December 2, 2020 / Accepted: February 16, 2021 / Published online: March 6, 2021

(C) The Author(s) 2021

\section{ABSTRACT}

Tele-ophthalmology is a rapidly evolving and exciting field that has become increasingly relevant amidst the current global pandemic. With advancements in technology, many components of an evaluation previously possible only in an office setting are becoming possible remotely, paving the way for at-home visits. Examples of these technologies include measurement of visual acuity using smartphones, remote monitoring of intraocular pressure using self-administered tonometers or implantable sensors, and use of digitally acquired slit-lamp or smartphone images for diagnosis of anterior segment pathology. While many examples showcasing the utility of telemedicine have been shown for diseases such as diabetic retinopathy, concerted efforts are needed to develop similar technology for anterior segment diseases. As technology continues to advance, it may be possible to diagnose and manage more anterior segment disease with telemedicine.

Keywords: Anterior segment; Cornea; COVID19; Global health; Telemedicine

\section{K. A. Alawa · C. S. Sales ( $\square)$}

Department of Ophthalmology, University of Iowa

Hospital and Clinics, Iowa City, IA, USA

e-mail: christopher-sales@uiowa.edu

\section{Key Summary Points}

Tele-ophthalmology is a rapidly evolving and exciting field that has become increasingly relevant amidst the current global pandemic.

Examples of tele-ophthalmology technologies applicable to the anterior segment include measurement of visual acuity using smartphones, remote monitoring of intraocular pressure using self-administered tonometers or implantable sensors, and use of digitally acquired slit-lamp or smartphone images for diagnosis of anterior segment pathology.

While many examples showcasing the utility of telemedicine have been shown for diseases such as diabetic retinopathy, concerted efforts are needed to develop similar technology for anterior segment diseases.

As technology continues to advance, it may be possible to diagnose and manage more anterior segment disease with telemedicine. 


\section{DIGITAL FEATURES}

This article is published with digital features, including a summary slide, to facilitate understanding of the article. To view digital features for this article go to https://doi.org/10.6084/ m9.figshare.14039708.

\section{INTRODUCTION}

Telemedicine, the remote delivery of medical services using technology, has been possible in its most basic form since the invention of the telegraph. As technology has advanced, so has the scope of medical care that can be offered remotely. Telemedicine within ophthalmology, or tele-ophthalmology, has been of interest to improve access to care and implement community screening, and in the current global pandemic, there is a large collective interest in leveraging tele-ophthalmology for at-home visits.

The key aspects of any ophthalmic evaluation include history, visual acuity, intraocular pressure (IOP), pupil examination, slit-lamp examination, and dilated fundus examination. Evaluation can be aided by diagnostic imaging such as external slit-lamp photographs, optical coherence tomography, fundus photography, and ultrasound. With advancements in technology, many components of an evaluation previously possible only in an office setting are becoming possible remotely. In this review, the current state of tele-ophthalmology within the cornea and anterior segment will be discussed. This article is based on previously conducted studies and does not contain any new studies with human participants or animals performed by any of the authors.

\section{HISTORY}

At the start of any visit, a comprehensive history is taken to document a patient's symptoms. While typically face-to-face in a clinic setting, history can be gathered by a variety of other means such as telephone, email, messaging within an electronic medical record (EMR), video chat, and more. There are many platforms available, both secure and insecure, that enable live synchronous audio/video communication. Caution should be taken when utilizing some of these technologies, as they must be compliant with the Health Insurance Portability and Accountability Act (HIPAA) to be used in a healthcare setting. The Center for Medicare and Medicaid Services (CMS) details a list of approved, HIPAA-compliant platforms for communication, and many institutions have internal guidelines as well.

\section{VISUAL ACUITY}

Measuring visual acuity involves reading a vision chart containing Snellen letters, tumbling E's, or other similar optotypes of known sizes and sufficient contrast placed at a known distance. While most visual acuity testing in clinic is done in long lanes and examination rooms, multiple studies have shown that visual acuity can be measured with reasonable accuracy and repeatability using smartphones and personal computers.

One such study evaluating a smartphone application for visual acuity measurement, "Peek Acuity," in adults with tumbling E optotypes found that it was comparable to standard clinical methods, although the test was not selfadministered and was instead done with the help of an examiner to facilitate testing at a distance [1]. Another study evaluating the same application in children aged 3-17 found that it was also comparable to standard clinical methods [2]. Another application, "Vision@Home," tested both distance acuity with the help of an examiner and self-administered near-acuity, and found that both were accurate [3]. In studies examining the use of personal computers for visual acuity measurement, many have shown that with a properly calibrated monitor and setup, visual acuity can be measured accurately [4].

Telemedicine technology can be used to test visual acuity in community-based settings and also allows patients to measure visual acuity at home. Caution should be taken, however, as there are numerous applications in the Apple 
and Android application stores that may not have been clinically validated and can therefore lead to erroneous visual acuity measurement. Another option provided by the American Academy of Ophthalmology (AAO) on their website is a printable vision chart with instructions detailing how patients can measure their visual acuity at home.

Although there may be some inter-test variability between different methods of visual acuity measurement, consistent use of the same method can be useful for at-home monitoring of visual acuity. Additional factors such as background brightness and ambient lighting should be considered, as non-optimal lighting may result in contrast inconsistencies that may skew visual acuity measurement. Most smartphones include an ambient light sensor, and some visual acuity smartphone applications adjust the brightness of the screen based on ambient lighting measurements to ensure consistent contrast.

\section{INTRAOCULAR PRESSURE}

The gold standard for IOP measurement is Goldmann applanation tonometry. Portable instruments such as the Tono-Pen and iCare tonometers are routinely used in clinics and have been shown to be comparable to Goldmann applanation. These instruments require a skilled technician as well as topical anesthesia (except for rebound tonometers such as the iCare) to obtain accurate measurements.

At-home IOP monitoring has been of interest to reduce clinic visits, chart diurnal variations in patient IOP, and to document IOP spikes that can be missed at regular clinic appointments. Multiple commercial devices are being developed and marketed for at-home IOP monitoring. These include the iCare HOME, a selfadministered rebound tonometry device, IOPsensing contact lenses such as Triggerfish, and implantable IOP sensors [5].

Studies comparing IOP measurements using the iCare HOME versus Goldmann applanation have shown that iCare HOME can underestimate IOP, but that it has reasonable agreement with Goldmann applanation [6]. IOP-sensing contact lenses offer a convenient method for athome IOP monitoring, but these have not been adopted for widespread clinical use due to their cost and because they measure in units of millivolts rather than millimeters of mercury [5]. Implantable IOP-sensors are being developed as well. Preliminary data suggest that these devices are safe and provide accurate IOP measurements; however, further investigation is needed before these devices can be widely adopted [5].

\section{EXAMINATION OF THE ANTERIOR SEGMENT}

Effective examination of the anterior segment requires a slit-lamp to obtain a high-magnification view and to highlight pathology using variable forms of illumination. Portable slitlamps have been used to examine patients who cannot use a regular slit-lamp, such as young children, or in areas without slit-lamps. In conjunction with a conventional slit-lamp examination, standard of care can often involve digital slit-lamp photographs to objectively document clinical findings for future comparisons.

A store-and-forward approach where testing or imaging is acquired and interpreted remotely has been applied to many medical specialties, and with sufficient image quality, the same could theoretically be applied to ophthalmology. One study attempted to evaluate this approach but exhibited mediocre results, finding that the diagnostic accuracy of standardized digital slit-lamp photographs for a wide range of clinical pathology was inferior to an in-person examination [7]. Poor diagnostic accuracy was attributed to a standardized approach to image acquisition, a strategy that stands in contradistinction to a targeted approach that would deliberately depict areas of concerning pathology. Poor accuracy was also thought to be related to the imaging system's inability to capture subtle findings, such as cell and flare.

An alternative strategy to the store-and-forward approach is acquiring images with a remotely operated slit-lamp. Rather than transmit slit-lamp images acquired by a photographer or a machine, one group created a 
mechanized slit-lamp with live audio/video and stereo-viewing capabilities for remote examination and diagnosis [8]. While not practical for at-home use, this system could be used at rural or ancillary sites where an ophthalmologist is not present. Furthermore, with artificial intelligence (AI) and other emerging technologies, an AI-based screening using an autonomous motorized slit-lamp for anterior segment disease might one day be on the horizon. While the current device appears to capture high-resolution video and provides a remote, interactive experience that closely mimics a traditional slitlamp examination, the system's diagnostic accuracy has yet to be studied.

In settings without access to a slit-lamp, there are other ways to acquire digital images with smartphones. With high-quality cameras available on most smartphones, external photographs of the eye are simple to obtain, and high-magnification anterior segment or fundus photographs can be captured with the use of external lenses [9-13]. One small study showed that epithelial defects were identifiable using a smartphone paired with a macro lens and cobalt blue filter [13]. Another study showed the clinical utility of quantitatively imaging corneal endothelial cells at subcellular resolution using a smartphone with a custom-made adapter and macro lens [14].

However, the preponderance of studies examining the diagnostic utility of anterior segment imaging with smartphones with or without macro lenses have generally shown inadequate sensitivity and specificity for clinical use $[15,16]$. Some of these limitations could be related to a lack of optical magnification, autofocus without the ability to refocus (most autofocus programs will identify the iris but not the cornea), and lack of a slit-beam. It may be worth repeating anterior segment telemedicine studies as both the software capabilities and smartphone hardware continue to improve.

\section{CONCLUSION}

Tele-ophthalmology is a rapidly evolving and exciting field that can help improve access to care and maintain patient and provider safety during the COVID-19 pandemic. Some aspects of the ophthalmic evaluation are possible remotely, and while many examples of its utility have been shown for diseases such as diabetic retinopathy, concerted efforts are needed to develop similar technology for anterior segment diseases. As technology continues to advance, it may be possible to diagnose and manage more anterior segment disease with telemedicine.

\section{ACKNOWLEDGEMENTS}

Funding. No funding or sponsorship was received for this study or publication of this article.

Authorship. All named authors meet the International Committee of Medical Journal Editors (ICMJE) criteria for authorship for this article, take responsibility for the integrity of the work as a whole, and have given their approval for this version to be published.

Disclosures. The authors, Karam A. Alawa and Christopher Sales, do not have any personal, financial, commercial, or academic conflicts of interest.

Compliance with Ethics Guidelines. This article is based on previously conducted studies and does not contain any new studies with human participants or animals performed by any of the authors.

Open Access. This article is licensed under a Creative Commons Attribution-NonCommercial 4.0 International License, which permits any non-commercial use, sharing, adaptation, distribution and reproduction in any medium or format, as long as you give appropriate credit to the original author(s) and the source, provide a link to the Creative Commons licence, and indicate if changes were made. The images or other third party material in this article are included in the article's Creative Commons licence, unless indicated otherwise in a credit line to the material. If material is not included 
in the article's Creative Commons licence and your intended use is not permitted by statutory regulation or exceeds the permitted use, you will need to obtain permission directly from the copyright holder. To view a copy of this licence, visit http://creativecommons.org/licenses/by$\mathrm{nc} / 4.0 /$.

\section{REFERENCES}

1. Bastawrous A, et al. Development and validation of a smartphone-based visual acuity test (peek acuity) for clinical practice and community-based fieldwork. JAMA Ophthalmol. 2015;133(8):930-7.

2. Zhao L, Stinnett SS, Prakalapakorn SG. Visual acuity assessment and vision screening using a novel smartphone application. J Pediatr. 2019;213:203210.e1.

3. Han X, et al. Development and validation of a smartphone-based visual acuity test (vision at home). Transl Vis Sci Technol. 2019;8(4):27.

4. Kumar S, Bulsara M, Yogesan K. Automated determination of distance visual acuity: towards teleophthalmology services. Clin Exp Optom. 2008;91(6):545-50.

5. Bhartiya S, et al. 24-hour Intraocular pressure monitoring: the way ahead. Rom J Ophthalmol. 2019;63(4):315-20.

6. Dabasia PL, Lawrenson JG, Murdoch IE. Evaluation of a new rebound tonometer for self-measurement of intraocular pressure. $\mathrm{Br} \mathrm{J}$ Ophthalmol. 2016;100(8):1139-43.
7. Kumar S, Yogesan K, Constable IJ. Telemedical diagnosis of anterior segment eye diseases: validation of digital slit-lamp still images. Eye (Lond). 2009;23(3):652-60.

8. Nankivil D, et al. Robotic remote controlled stereo slit lamp. Transl Vis Sci Technol. 2018;7(4):1-1.

9. Mohammadpour M, Mohammadpour L, Hassanzad M. Smartphone assisted slit lamp free anterior segment imaging: a novel technique in teleophthalmology. Cont Lens Anterior Eye. 2016;39(1):80-1.

10. Ludwig CA, et al. Training time and quality of smartphone-based anterior segment screening in rural India. Clin Ophthalmol. 2017;11:1301-7.

11. Kaya A. Ophthoselfie: detailed self-imaging of cornea and anterior segment by smartphone. Turk J Ophthalmol. 2017;47(3):130-2.

12. Chiong HS, Fang JL, Wilson G. Tele-manufactured affordable smartphone anterior segment microscope. Clin Exp Optom. 2016;99(6):580-2.

13. Maamari RN, et al. Novel telemedicine device for diagnosis of corneal abrasions and ulcers in resource-poor settings. JAMA Ophthalmol. 2014;132(7):894-5.

14. Toslak D, et al. Smartphone-based imaging of the corneal endothelium at sub-cellular resolution. J Mod Opt. 2017;64(12):1229-32.

15. Woodward MA, et al. Teleophthalmic approach for detection of corneal diseases: accuracy and reliability. Cornea. 2017;36(10):1159-65.

16. Nagra M, Vianya-Estopa M, Wolffsohn JS. Could telehealth help eye care practitioners adapt contact lens services during the COVID-19 pandemic? Cont Lens Anterior Eye. 2020;43(3):204-7. 